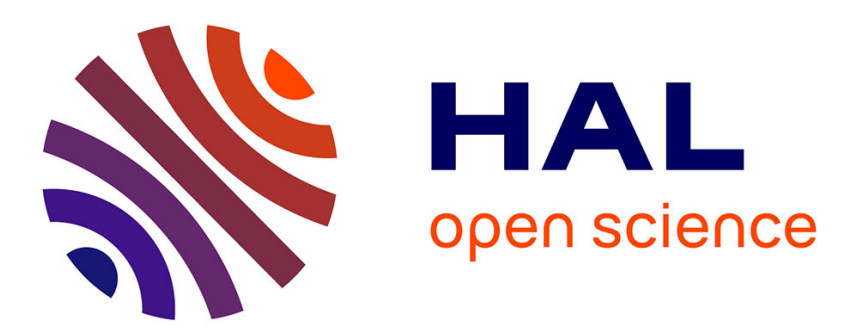

\title{
Le faciès monétaire de Villeneuve-Saint-Germain et ses éléments de datation
}

Jean Debord

\section{To cite this version:}

Jean Debord. Le faciès monétaire de Villeneuve-Saint-Germain et ses éléments de datation. Gallia Archéologie de la France antique, 1995, Les potins gaulois, 52, pp.61-78. 10.3406/galia.1995.3124 . hal-01911256

\author{
HAL Id: hal-01911256 \\ https://hal.science/hal-01911256
}

Submitted on 27 Feb 2020

HAL is a multi-disciplinary open access archive for the deposit and dissemination of scientific research documents, whether they are published or not. The documents may come from teaching and research institutions in France or abroad, or from public or private research centers.
L'archive ouverte pluridisciplinaire HAL, est destinée au dépôt et à la diffusion de documents scientifiques de niveau recherche, publiés ou non, émanant des établissements d'enseignement et de recherche français ou étrangers, des laboratoires publics ou privés.

\section{(ㅇ)(1) $\$$}

Distributed under a Creative Commons Attribution - NonCommercial - NoDerivatives| 4.0 


\title{
3.4. Le faciès monétaire de Villeneuve-Saint-Germain et ses éléments de datation
}

\section{Jean Debord}

\begin{abstract}
Résumé. Le faciès monétaire de Villeneuve-Saint-Germain est caractérisé par une forte dominante des monnaies en potin (81,79\%). Le monnayage des Suessiones représente $84,20 \%$ de l'ensemble dont 73,76\% sont issus de l'atelier monétaire local. Les éléments de datation sont fournis principalement par les fibules et les amphores, mais aussi par les parures en verre, la céramique, différents objets d'importation... L'ensemble du mobrilier s'intègre parfaitement dans la phase de La Tène. D2. Quelques éléments de datation absolue semblent indiquer une occupation postérieure à la guerre des Gaules. La comparaison avec l'oppidum voisin de Pommiers s'avère peu probante par le. seul fait que ce dernier site n'est connu que par des ramassages de surface. Seules les fouilles récentes sont exploitables, mais elles demeurent très ponctuelles n'ayant porté que sur une coupe du rempast et $150 \mathrm{~m}^{2}$ à l'intérieur de l'enceinte.
\end{abstract}

\begin{abstract}
Zusammenfassung. Die in Villeneuve-Saint-Germain gefundenen Münzen zeichnen sich durch eine slarke Dominante an Potinmünzen $(81,79 \%)$ aus. Die. Münzenproduktion der Suessiones machen $84,20 \%$ der Gesamtherstellung aus, wovon 73,76\% aus der Lokalmünzstätte stammt. Die Datierungselemente beruhen grösstenteils auf den Filben und Amphoren, sowie dem Glasschmuck, der Keramik und andem importierten Gegenständen... Das gesamle Fundmaterial lässt sich perfekt in die La Tène D2-Phase integrieren. Einige absolute Datierungselemente scheinen auf eine, den gallischen Kriegen folgende Beselzung schliessen zu lassen. Der Vergleich mit dem benachbarlen Oppidum von Pommiers stellt sich als wenig beweiskräftig heraus, da diese letztere Fundort nur an der Oberfläche untersucht wurde. Einzig die neulich unternommenen Ausgrabungen können zur Auswertung benutzt werden. Diese Untersuchung beschränkt sich jedoch auf einen Ausschnitt des Walls sowie auf weitere 150 Quadratmeter der Innenfläche.
\end{abstract}

Oppidum à fleur d'eau s'inscrivant dans un méandre de l'Aisne, le site de Villeneuve-Saint-Germain a fait l'objet de fouilles entre 1973 et 1984 . Ces recherches ont permis d'exhumer sept cent quarante-sept monnaies gauloises et de mettre en évidence la présence d'un atelier monétaire qui est à l'origine de monnaies en potin et en argent (Debord, 1989).

\section{LE FACIÈS MONÉTAIRE}

Ce qui frappe tout d'abord à l'examen de ce corpus monétaire, c'est la très forte dominante des pièces en potin par rapport aux autres métaux. Le bronze coulé représente, en effet, $81,79 \%$ du nombre des individus contre $9,64 \%$ pour le bronze frappé, $8,43 \%$ pour l'argent et seulement $0,13 \%$ (un exemplaire) pour l'or (fig. 21,1 ). Si l'on se réfère au nombre de types (qui est de 44 ), 45,45\% sont en potin, 36,36\% en bronze frappé, $15,91 \%$ en argent et $2,27 \%$ en or (fig. 21, 2). Si l'on examine l'origine des monnaies (fig. 21, 3), 84,20\% des exemplaires sont attribuables au Suessiones, 73,76\% étant issus de l'atelier monétaire local. Seulement $11,11 \%$ proviennent d'autres peuples. Les $4,69 \%$ restants sont des monnaies non attribuées ou non identifiables. Les monnaies exogènes se répartissent ainsi (fig. 22) : quarante-six exemplaires $(6,16 \%)$ appartiennent aux Remi, sept exemplaires $(0,94 \%)$ aux Senones, sept exemplaires aux Nervii, six exemplaires $(0,80 \%)$ aux Carnutes, quatrc cxemplaires (0,54\%) aux Ambiani, la même proportion est attribuable aux Parisii, trois exemplaires $(0,40 \%)$ aux Veliocasses, deux exemplaires $(0,27 \%)$ aux Sequani, les Leuci, les Eburovices, les Turones et la Bretagne insulaire étant représentés chacun par un exemplaire. Ce sont donc les Suessiones et les Remi qui constituent l'essentiel du monnayage de Villeneuve-Saint-Germain. I a présence, en quantité notable, de monnaies rèmes (en majorité des potins) ne saurait surprendre puisqu'on sait qu'après la défaite de Galba, en 57 avant J.-C., César a placé les Suessiones sous l'autorité des Remi (B.G. VIII,6,2). On peut y voir un indice de datation tardive du site puisqu'il semble bien qu'avant la guerre avec Rome, si l'on se réfère au monnayage, il n'y eut que peu de contacts entre les deux peuples. Le statère anépigraphe des Suessiones, par exemple, dont la création est à mettre en relation avec la formation de l'armée confédérée dont Galba devait prendre le commandement (Scheers, 1977, p. 60 sq.) est connu par de nombreux exemplaires et la concentration des trouvailles désigne bien le territoire suession comme 

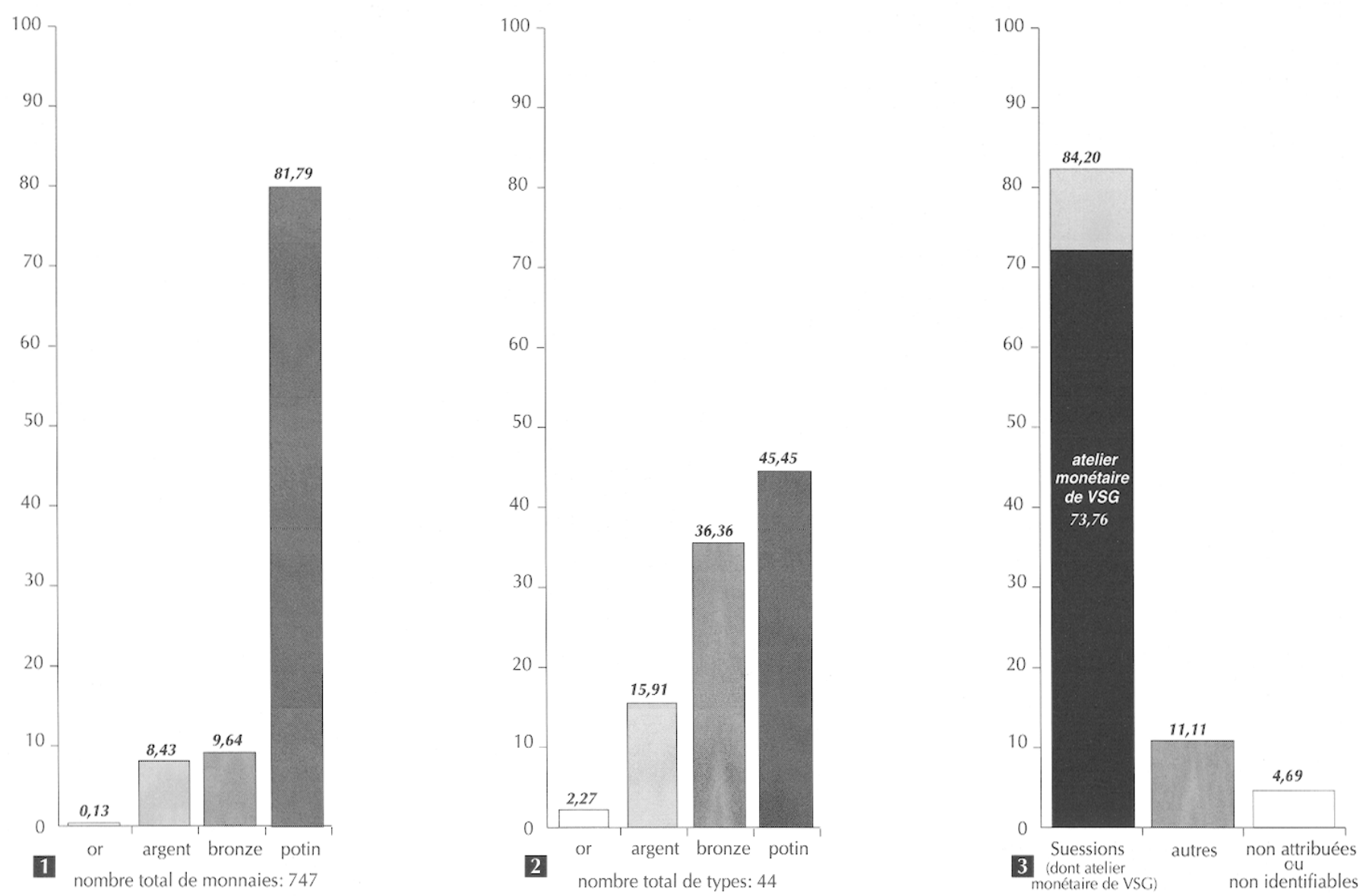

Fig. 21. Faciès monétaires de Villeneuve-Saint-Germain.

1. faciès des monnaies par composition métallique;

2. faciès des lypes par composition métallique;

3. faciès des monnaies par peules.

centre de dispersion de ce numéraire. Or, la distribution de ces monnaies fait apparaitre deux axes privilégiés, l'un en direction de la Bretagne insulaire, l'autre vers le centre de la Gaule alors qu'un seul point de découverte cst connu chcz les Remi (Debord, 1985). De même, pour le monnayage trimétallique si nombreux au nom de CRICIRV, deux points de découverte seulement apparaissent dans la Marne sur la carte de répartition établie par Simone Scheers (1977, p. 379).

Comme le montrent très clairement les cartes (fig. 2326), les espèces en potin issues de l'atelier monétaire n'ont guère dépassé les limites du pays suession. Quant aux espèces en argent, sans doute l'ultime monnayage de ce peuple (Debord, 1987b), elles n'ont pratiquement pas circulé hors de leur lieu d'émission.
Description sommaire des potins du site de VilleneuveSaint-Germain

\section{Potins attribuables aux Suessiones}

Type Scheers 185, classe III (LT XXX, 7458, variante) : cent quatre-vingt-trois exemplaires (fig. 23). La face qui est généralement considérée comme le droit montre, dans un cercle de grènetis, deux quadrupèdes affrontés. $\mathrm{Au}$ revers, deux sangliers affrontés sont facilement identifiables par leurs soies dressées sur le dos. Grènetis au pourtour. La forte proportion de cette monnaie sur le site incite à y voir une production locale.

Type Scheers 185, classe I (LT XXX, 7467) : deux exemplaires. Sur l'avers se voient deux chèvres affrontées de part et d'autre de la légende AGHD. Grènetis périphérique. Au revers figurent un loup et un sanglier opposés dans un cercle de grènetis. L'attribution traditionnelle de cette monnaie aux Senones était fondée sur une interprétation abusive de la légende lue comme une abréviation d'« AGED (INCUM SENONUM) ». Elle est insou- 


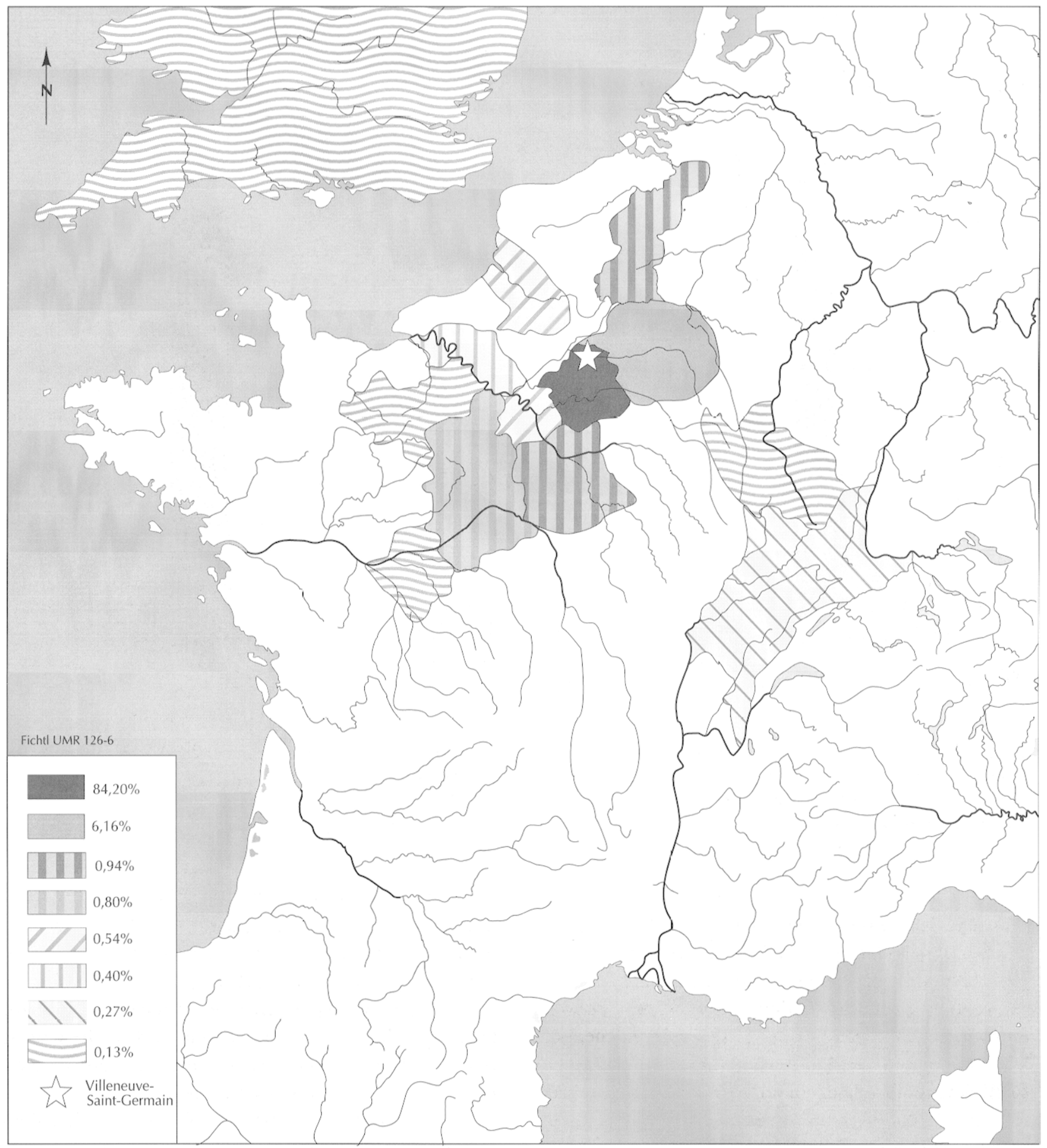

Fig. 22. Provenance des types monétaires en potin trouvés à Villeneuve-Saint-Germain. 


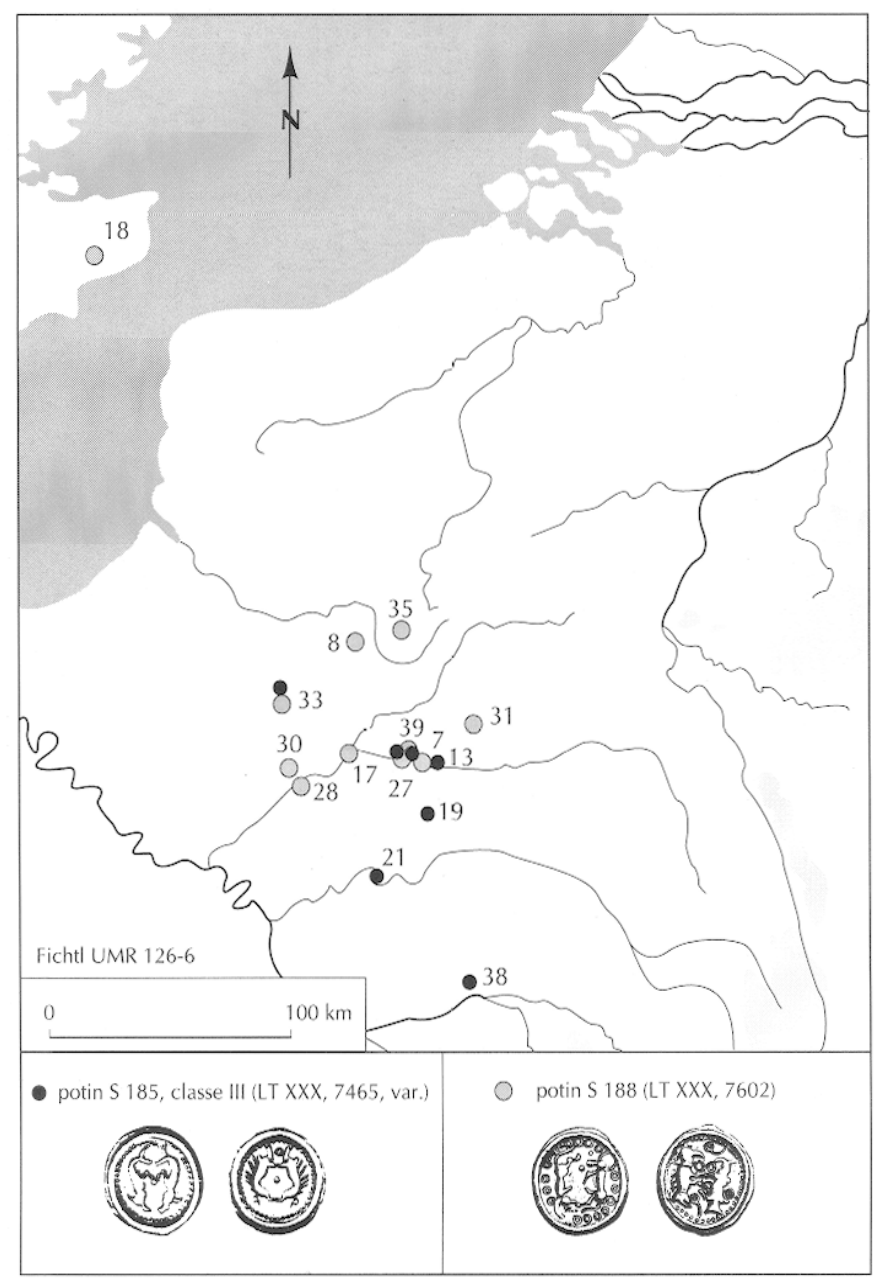

Fig. 23. Carte de diffusion des potins Scheers 185, classe III, et Scheers 188.

tenable, les trouvailles étant principalement concentrées en pays bellovaque et suession. Si la classe III, très fortement représentée, peut être considérée comme étant issue de l'atelier monétaire local, il n'en va pas de même pour ce potin épigraphe représenté par deux exemplaires seulement.

Type Scheers 185, classe II (IT XXX, 7458) : sept exemplaires. Au droit, deux chèvres affrontées dans un cercle de grènetis. Au revers, un loup et un sanglier sont opposés de part et d'autre d'un globule central. L'attribution traditionnelle aux Senones appelle les mêmes remarques que précédemment.

Type Scheers 188 (LT XXX, 7602) : neuf exemplaires (fig. 23). Au droit, deux taureaux sont disposés tête-bêche dans un cercle d'annelets. Au revers, on voit deux oiseaux affrontés avec quelques annelets dans le champ et un grènetis périphérique. C'est la découverte d'un exemplaire

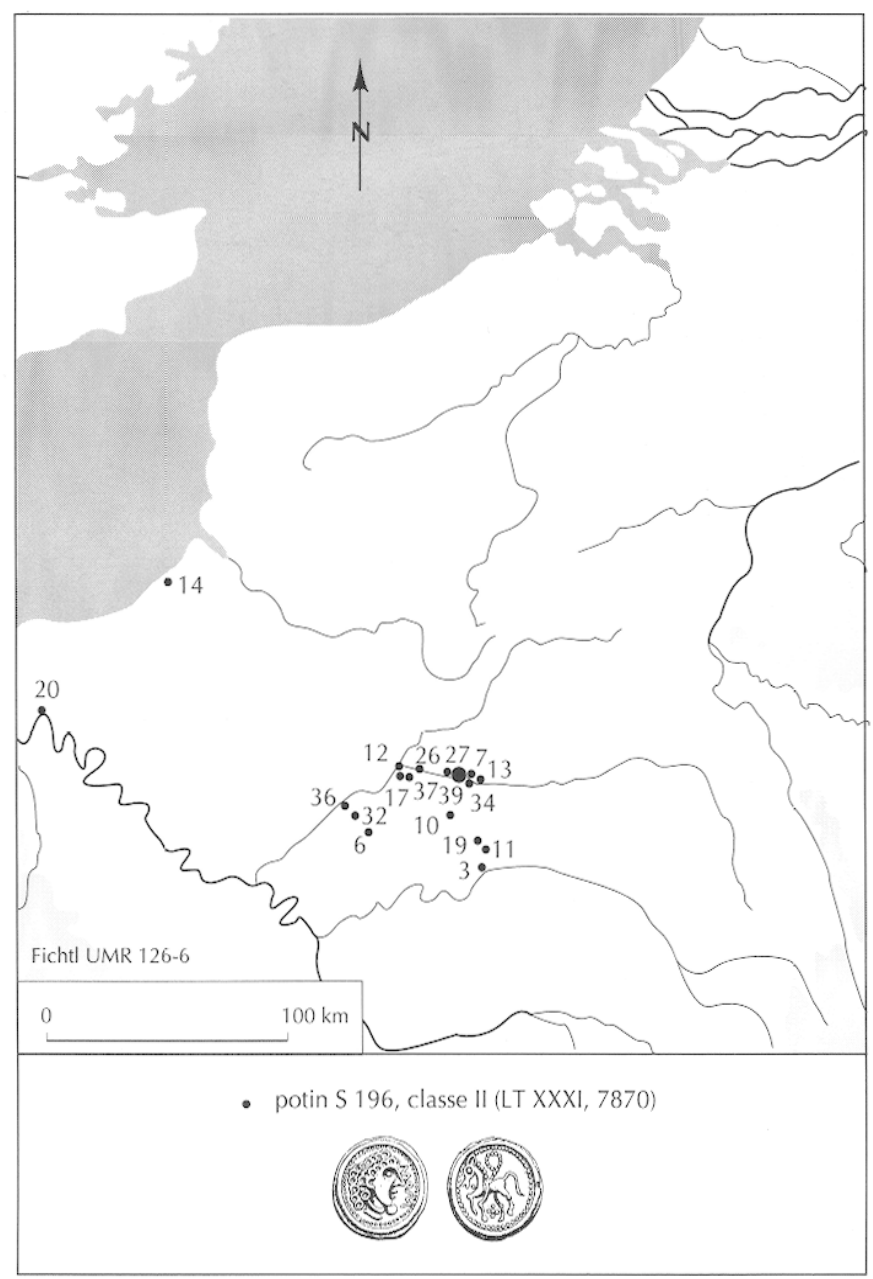

Fig. 24. Carte de diffusion des potins Scheers 196, clusse II.

mal venu à la coulée (Debord, Ilett-Fleury, 1983) qui atteste la fabrication locale de cette monnaie relativement rare qui était traditionnellement donnée aux Meldi.

Type Scheers 196, classe II (IT XXXI, 7870) : cent trente et un exemplaires (fig. 24). L'avers montre une tête à droite dont la chevelure abondante est figurée par une triple rangée d'annelets. Une ligne en zigzag est placée devant le visage. Entourage de grènetis. Sur l'autre face, on voit un cheval à gauche dont la crinière perlée se termine au-dessus du dos par un annelet centré. Sous le cheval, cinq points sont disposés en croix. Grènetis périphérique. La présence de cinq déchets de coulée montre bien que c'est l'atelier monétaire de Villeneuve-SaintGermain qui est à l'origine de ce type.

Type Scheers 196, classe I (LT XXXI, 7862) : seize exemplaires. Au droit, un cercle d'annelets entoure une tête à droite dont la chevelure est rendue par deux ran- 


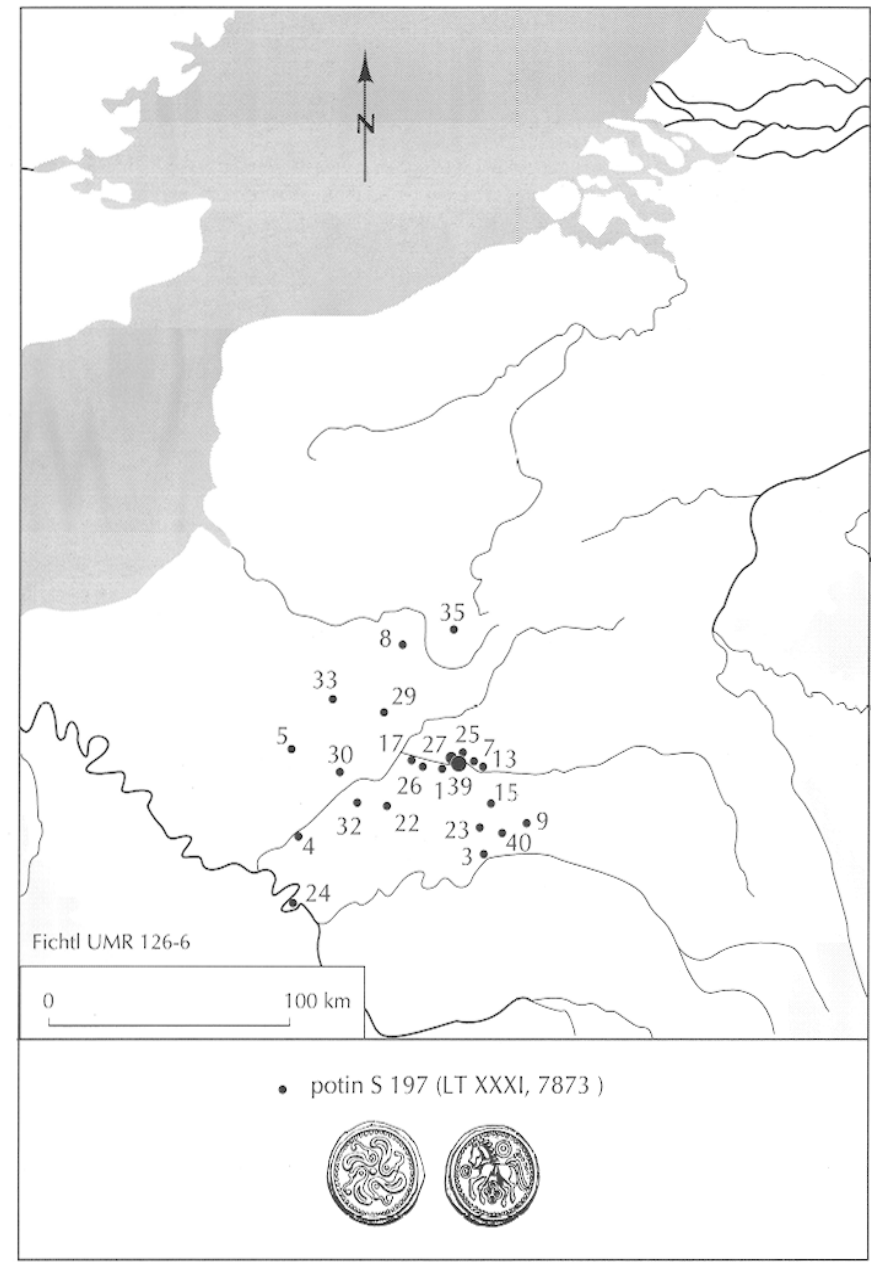

Fig. 25. Carte de diffusion des potins Scheers 197.

gées de globules. Au revers, un cheval à crinière perlée galope à gauche dans un entourage d'annelets et de points. Sous le cheval se trouve un $\mathrm{V}$ renversé dont chaque branche se termine par un annelet. En haut, un signe en forme de $S$, placé horizontalement, a l'une de ses extrémités terminée par un globule, l'autre par un annelet. Traditionnellement attribuée aux Silvanecti, cette monnaie coexiste généralement sur les sites avec LT 7870 et LT 7859. Simone Scheers (1977, p. 173) considère qu'elles forment les trois classes d'une même série. L'aire de dispersion de ce type semble situer son origine chez les Suessiones, mais le nombre relativement peu élevé d'individus en provenance de notre site ne permet pas d'affirmer qu'il s'agit d'une production locale.

Type Scheers 196, classe III (LT XXXI, 7859) : quatre exemplaires. Sur l'avers est figurée une tête à droite dans un cercle de grènetis. La chevelure est rendue par une

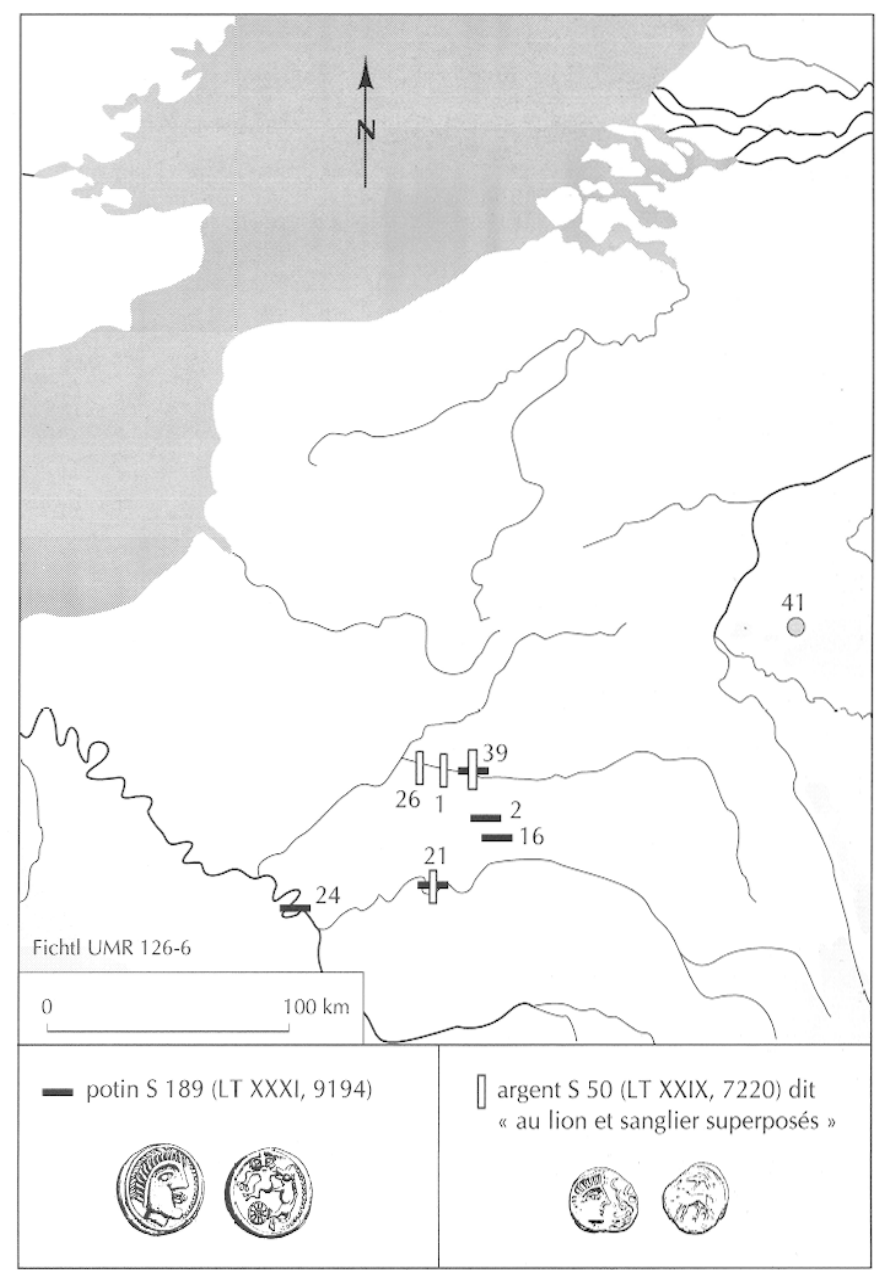

Fig. 26. Carte de diffusion des potins Scheers 189.

série de traits courbes et parallèles. Au revers, un cheval galopant à gauche est surmonté d'un annelet centré. Grènetis périphérique. Si les trouvailles de cette monnaie sont concentrées en pays suession, elle n'est que faiblement représentée à Villeneuve-Saint-Germain. Mais elle présente une grande affinité typologiquc avcc dcs monnaies en argent issues de cet atelier, notamment avec la monnaie dite " au bige ", tant par le droit que par le revers (Debord, 1980, p. 43) et avec LT 7220 par le droit. Aussi ne semble-t-il pas invraisemblable de lui accorder la même origine.

Type Scheers 197 (LT XXXI, 7873) : cent soixantetreize exemplaires (fig. 25). Le droit présente un élégant fleuron dérivé du svastika. Entourage de grènetis. Le revers est orné d'un cheval harnaché passant à gauche, surmonté d'un annelet centré. Un autre annelet centré, plus petit, est placé devant le poitrail. Sous le ventre se 
voit un motif formé de quatre petits cercles disposés en croix (ou de cinq globules sur certains exemplaires). Entourage de petits traits obliques. Outre le nombre important de pièces recueillies, un raté de fabrication vient témoigner de l'origine locale de cette monnaie.

Type Scheers 189 (LT XXXI, 9194) : un exemplaire (fig. 26). Le droit montre une tête à droite dont la chevelure cst figuréc par deux rangées de petits traits courbes et parallèles. Un grènetis occupe le bord de la pièce devant le visage. Au revers, un loup gigantesque dévore le bras d'un personnage moustachu vu de face. Une roue occupe l'espace laissé libre entre le personnage et les pattes postéricures du fauve. Il n'est connu que quelques exemplaires de cette monnaie. C'est l'homotypie du droit avec des monnaies en argent provenant de l'atelier de Villeneuve-Saint-Germain qui nous incite à accorder une origine commune à ces différents numéraires (Debord, 1993a).

Type Scheers 198 (LT XXXII, 7905) : huit exemplaires. Une tête à droite, très stylisée, occupe l'avers. Au revers, un sanglier stylisé surmonte un grand annelet centré qui occupe le centre du flan. Derrière l'animal se voit un petit cercle centré tandis que le bas de cette face montre un torque ouvert encadrant cinq globules. Les trouvailles de ce type se concentrent aux confins des pays bellovaque et suession, de telle sorte qu'il apparaît difficile d'en attribuer le mérite à l'un de ces deux peuples de préférence à l'autre. Toutefois, des découvertes récentes (Villeneneuve-Saint-Germain, Condé-sur-Aisne, Chassemy...) apportent de nouveaux arguments en faveur d'une attribution aux Suessiones.

\section{Potins attribuables aux Remi}

Type Scheers 191 (LT XXXII, 8124) : neuf exemplaires. Le droit présente un personnage à chevelure nattée marchant à droite et tenant un torque d'une main, une lance (?) de l'autre. Sur l'autre face, un animal à droite semble dévorer une proie. Il est surmonté d'un signe serpentiforme. La date d'apparition de ce potin fait actuellement l'objet d'une polémique entre partisans d'une chronologie haute et ceux d'une datation basse. Dans un article à paraître, nous étudions les monnaies trouvées en fouilles à Condé-sur-Suippe au cours de la campagne 1987 et dont l'essentiel du corpus est constitué par ce type. Nous tentons de montrer que rien ne semble, actuellement, justifier une datation très antérieure à la guerre des Gaules. Cette monnaic apparaît fréquemment dans des contextes qui sont datables antérieurement à 60 avant J.-C. par les autres mobiliers archéologiques. Mais il s'agit là du contexte général et, lorsque les fouilleurs ont donné des précisions quant à la situation exacte de la monnaie en question, il apparait qu'elle est toujours située dans une couche tardive qui marque la fin de l'occupation du site. Or, la facilité de fabrication d'un monnayage en potin fait qu'il a pu être mis en circulation, en quantité importante, dans des délais très courts et que sa présence peut ne correspondre qu'à une période de circulation très brève. Seules les découvertes dans des ensembles clos, c'est-àdire des sépultures, pourraient apporter des éléments de datation incontestables. Nous ne tiendrons pas compte ici de l'association supposée d'un potin de ce type avec du matériel de la Tène C2 dans une incinération trouvée au début du siècle près de Mayence (Polenz, 1982), l'homogénéité du mobilier de la tombe ayant été mise en doute dès la publication. En revanche, la publication récente des séries de potins trouvés sur l'habitat gaulois d'AcyRomance (Lambot, Delestrée, 1991) fait état de la découverte de huit potins I.T 8124 dans la tombe à incinération I 104 que les auteurs veulent dater du II' $\mathrm{s}$. avant J.-C., vers 130/120. Ies principaux éléments de datation retenus sont la présence d'un bracelet en verre bleu à section en $\mathrm{D}$ et de fibules de Nauheim. Le bracelet appartient au groupe Haevernick $3 \mathrm{~b}$ (bleu, à section en $\mathrm{D}$, avec décor ondé jaunc). Or, dans un article récent consacré à "La parure celtique en verre en Europe centrale ", Natalia Venclova (1989) a bien montré que si de tels bracelets ont été découverts dans des sépultures de La Tène $\mathrm{Cl}$, ils réapparaissent à La Tène $\mathrm{D}$ et " qu'il ne semble y avoir aucune relation, ni dans le temps ni dans l'espace " entre ces productions (p. 89). Le même auteur souligne (p. 88) qu'il n'est pas exceptionnel de rencontrer des modèles anciens dans un contexte plus récent, certains types, parmi les plus populaires, ayant été fabriqués pendant une longue période. Quant aux fibules du type de Nauheim, on sait que leur fabrication s'est étalée sur une longue durée et qu'elles ne sauraient être retenues comme indice précis de datation d'un site. En conclusion, il nous semble que, dans un souci de gommer toute trace de subjectivité, l'on ait peut-être accordé trop d'importance aux éléments statistiques qui n'ont pas toujours la signification qu'on veut leur donner, la durée de circulation d'un monnayage pouvant être sans commune mesure avec celle de l'occupation du site.

Type Scheers 194 (IT XXXII, 8145) : quatorze exemplaires. Le droit est occupé par un personnage assis en 
pose bouddhique et tenant un torque de la main droite, une tresse de ses cheveux de la main gauche. Le revers portc un sanglicr à droitc surmonté d'un signe serpentiforne devant lequel figure un astre. Un second astre est placé entre les pattes de l'animal.

Type Scheers 195 (IT XXXII, 8351) : dix exemplaires. On voit, au droit, un bucrâne encadré de deux motifs en forme de $S$ et surmonté d'annelets centrés. Au revers, un animal à droite dévore une proie, très proche de celui figurant sur Scheers 191. L'aire de dispersion des trois potins que nous venons de décrire est sensiblement la même.

\section{Potins attribuables aux Nervii}

Type Scheers 190, classe III, dite " rameau B » (IT XXXV, 8636) : quatre exemplaires. Au droit figure le motif " au rameau " constitué de huit mèches ondulées réparties de part et d'autre d'une tige perlée. Grènetis périphérique. Au revers, un cheval gauche dans un cercle d'annelets.

Type Scheers 190, classe IV, dite "rameau A " (LT XXXV, 8620) : trois exemplaires. Au droit, rameau d'exécution beaucoup plus grossière que sur le type précédent. Au revers, cheval à droite. Le monnayage " au rameau » forme un ensemble complexe dont l'attribution de certaines classes reste actuellement en suspens.

\section{Potins attribuables aux Senones}

Type LT XXX, 7405 : un exemplaire. L'avers montre une tête casquée à droite et le revers un cheval galopant à gauche, la queue relevée au-dessus du dos.

Type LT XXX, 7417 : un exemplaire. Sur l'avers est figurée une tête à droite à chevelure hirsute indiquée par une série de traits parallèles. Au revers, un cheval passe à gauche, la queue relevée au dessus du dos. Globules dans le champ. L'attribution traditionnelle de ces deux types aux Senones n'est pas certaine. Les trouvailles récentes semblent plutôt indiquer une origine chez un peuple riverain de la Seine (Delestrée, 1985, p. 55).

\section{Potins attribuables aux Parisii}

Type IT XXXI, 7820 : deux exemplaires. Le droit présente une tête casquée à droite. Le revers est orné d'un cheval galopant à gauche dans un cercle de grènetis. L'attribution de ce numéraire aux Parisii est incertaine.

\section{Potin attribuable aux Leuci}

Type LT XXXVII, 9180 : un exemplaire. On voit au droit une tête à gauche. Au revers, un sanglier à gauche entre les pattes duquel se trouve un crâne humain.

\section{Potins attribuables aux Carnutes}

Type LT XIX, 6188 : deux exemplaires. Sur l'avers, tête à droite à la chevelure abondante. Au revers, animal à gauche au poil hérissé, la langue pendante. Une étoile se trouve sous les pattes antérieures, une autre sous le ventre de l'animal.

\section{Potin attribuable aux Turones}

Type BN 5674, dit potin «à la tête diabolique » : un exemplaire. Au droit, tête à gauche, très schématisée, l'œil étant figuré par une cavité large et profonde. Au revers, animal à gauche, la queue redressée au-dessus du dos.

\section{LES ÉLÉMENTS DE DATATION}

\section{Les fibules (fig. 27)}

Au nombre de quatre cent une, ce sont elles qui constituent l'indice chronologique le plus fiable.

Type Feugère 8 ("fibules à ailettes naissantes", « Knotenfibeln », «Almgren $65 »)$ : ce type est représenté par vingt-sept exemplaires, soit $6,73 \%$. Un accord général semble se faire autour d'une datation au troisième quart du I ${ }^{\text {er }}$ s. avant J.C. Michel Feugère (1985, p. 238) remarque qu'en cumulant les provenances des fibules du type 8a (ressort à quatre spires et corde interne), les plus nombreuses, on obtient un faciès qui est celui de l'extrême fin de La Tène.

Type Feugère 7c (fibules " à coquille ", "Schüsselfibeln ") : trente-cinq exemplaires, soit $8,73 \%$. Que ce soit à Bâle, à Manching ou à Roanne, ces fibules se trouvent dans des couches datées de la seconde moitié du $\mathrm{I}^{\text {er }}$ s. avant J.-C. Les exemplaires de Villeneuve-SaintGermain sont, soit de petites fibules en bronze, soit, pour le plus grand nombre, de grandes fibules en fer à arc triangulaire rubané venant couvrir le ressort à quatre spires et corde interne. Ces grandes fibules en fer couvrent principalement les rëgions de Trèves, de Luxembourg et du 


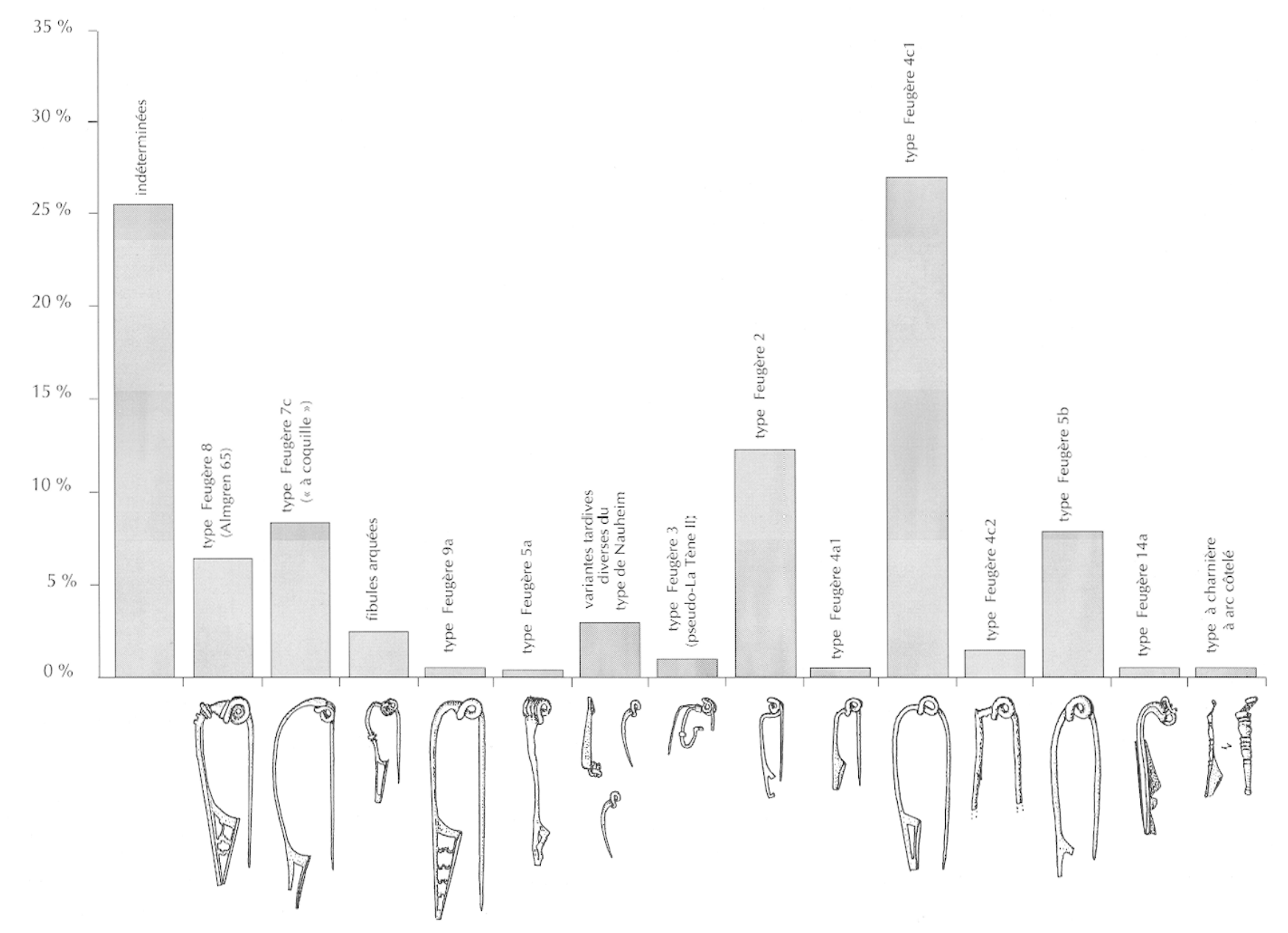

Fig. 27. Types de fibules trouvés à Villeneuve-Saint-Germain.

bassin de Neuwied où elles semblent apparaître au dernier tiers du $\mathrm{I}^{\text {er }}$ s. avant J.-C. jusqu'à la période augustéenne tardive ${ }^{36}$.

Fibules arquées ( Geschweiftefibeln ») : représentées ici par dix exemplaires $(2,49 \%)$, ce type est représentatif de l'horizon Bâle, Cathédrale, c'est à dire Ia Tène D2 (Furger-Gunti, 1979).

Type Feugère $9 a$ : deux exemplaires $(0,50 \%)$. Bien représenté dans le sud de la France où il apparaît souvent dans des contextes tardifs, ce type est cependant signalé sur quelques sites plus septentrionaux. A. Furger-Gunti (1979, p. 128) a publié un exemplaire provenant des fossés d'Alésia, ce qui ramène le terminus post quem vers la fin de la première moitié $d u \mathrm{I}^{\mathrm{er}} \mathrm{s}$. avant notre ère. Il peut donc être daté approximativement entre -60 et -10, mais sa pré-

36. Renseignements aimablement communiqués par Barbara Jürgens que nous remercions. sence sur de nombreux sites tardifs tendrait à montrer que c'est surtout vers la fin du siècle qu'il a été utilisé.

Type Feugère 5a : un seul exemplaire, soit 0,25\%. Il s'agit de la fibule de Nauheim classique à arc triangulaire. Ce type est caractéristique de l'horizon Bâle, Usine à gaz, c'est à dire de La Tène D1. Il perdure à La Tène D2 sur la colline de la Cathédrale où il est représenté par quelques exemplaires. La présence à Villeneuve-SaintGermain d'une seule fibule de ce type n'est donc pas significative et ne saurait être retenue comme indice de datation.

Variantes (tardives) diverses du type de Nauheim : onze exemplaires $(2,74 \%)$. Ce sont de petites fibules en fer, à corde interne ou externe, élaborées selon le schéma de la fibule de Nauheim classique. Leur arc triangulaire présente toujours une courbure très prononcée qui les rapproche du type Feugère $4 \mathrm{a} 2$ daté postérieurement à Tibère (Feugère, 1985, p. 203), ce qui nous incite à pro- 
poser pour ces variantes une datation tardive au sens large, sans qu'il soit possible d'être plus précis. L'un des onze exemplaires est une ébauche qui pourrait correspondre à une fibule du type Feugère $5 \mathrm{a}$ abandonnée en cours de fabrication (Debord, 1993b, p. 96, $\mathrm{n}^{\circ} 6$ ).

Type Feugère 3 (pseudo-La Tène II) : trois exemplaires $(0,75 \%)$ dont l'un, en fer, appartient au type $3 \mathrm{bl}$ (corde interne), les deux autres (l'un en fer, l'autre en bronze) au type $3 c$ (corde externe). Selon Michel Feugère, le type $3 \mathrm{~b} 1$, présent sur de nombreux sites dans toute la Gaule, n'apparaîtrait qu'à la fin du règne d'Auguste et l'on pourrait, pour le type 3c, proposer une datation analogue (1985, p. 196-197).

Type Feugère 2 : cinquante-deux exemplaires $(12,9 \%)$.

'lype Feugère $4 \mathrm{al}$ : un exemplaire $(0,25 \%)$.

Type Feugère $4 \mathrm{cl}$ : cent treize exemplaires $(28,18 \%)$.

Type Feugère 4c2 : six exemplaires $(1,50 \%)$.

Les quatre types précités sont des fibules filiformes qui ne constituent pas un repère chronologique très précis. Présentes sur de nombreux sites, elles abondent principalement durant les deux derniers tiers du I ${ }^{\text {er }}$ s. avant J.-C. (Feugère, 1978, p. 154).

Type Feugère $5 b$ : trente-trois exemplaires $(8,2 \%)$ dont un seul en bronze. Michel Feugère assimile aux fibules de Nauheim ce type filiforme à ressort à quatre spires et corde interne dont l'arc ne présente pas de courbure prononcée et se poursuit presque rectiligne jusqu'au porte-ardillon ajouré. Il s'agit presque toujours de fibules en bronze. On note, toutefois, quelques exemplaires en fer, notamment à Vienne, colline SainteBlandine (Chapotat, 1970). Sur notre site, la quasi-totalité est en fer. Là encore la fourchette chronologique est large et l'on peut proposer, comme pour les types précédents, une datation couvrant les deux derniers tiers du $\mathrm{I}^{\mathrm{er}}$ s. avant J.C.

Type Feugère 14a : un exemplaire $(0,25 \%)$. Ce genre de fibule est largement répandu et se trouve sur de nombreux sites tels que Pommiers, Gergovie, Bibracte... Bernard Lambot en a publié treize exemplaires provenant de la forêt de Compiègne (1975, p. 21). Il s'agit d'un type que l'on ne saurait faire remonter au-delà du troisième quart du $\mathrm{I}^{\mathrm{er}} \mathrm{s}$. avant notre ère.

Type à charnière à arc côtelé : cet exemplaire a été trouvé en surface des labours et ne peut donc être utilisé pour la datation du site gaulois. C'est un type galloromain apparu durant la seconde moitié du $\mathrm{I}^{\mathrm{er}} \mathrm{s}$. de notre ère et qui couvre le $\mathrm{II}^{\mathrm{e}}$ et une partie du $\mathrm{III}^{\mathrm{e}} \mathrm{s}$.

\begin{tabular}{|c|c|c|}
\hline TYPE & HAUTEUR & INCLINAISON \\
\hline 1 & $<35 \mathrm{~mm}$ & $49-70^{\circ}$ \\
\hline 2 & $34-45 \mathrm{~mm}$ & $55-80^{\circ}$ \\
\hline 3 & $40-55 \mathrm{~mm}$ & $>80^{\circ}$ \\
\hline 4 & $>55 \mathrm{~mm}$ & $>80^{\circ}$ \\
\hline
\end{tabular}

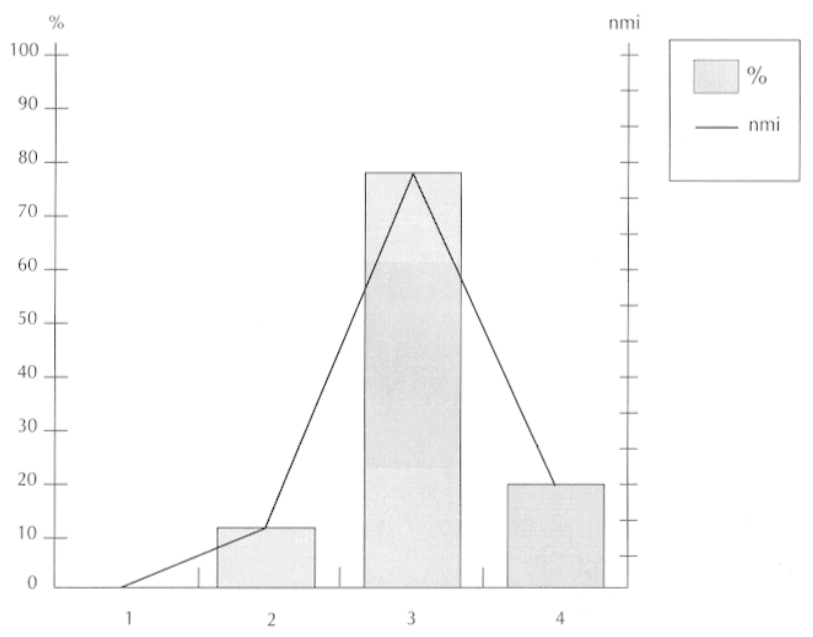

Fig. 28. Évolution de la hauteur et de l'inclinaison de la lèrre des amphores. (Diagramme d'après Bénédicte Hénon).

Fibules indéterminées : cent cinq exemplaires, il peut s'agir soit de fibules filiformes incomplètes dont il n'est pas possible de déterminer la position de la corde ou la courbure de l'arc (ce qui interdit un classement dans un type précis, mais nous avons vu que l'ensemble des fibules filiformes peut être situé dans les deux derniers tiers du $\mathrm{I}^{\mathrm{er}}$ s. avant J.-C.), soit de fragments trop oxydés ou trop petits pour permettre une détermination. L'ensemble des fibules de Villeneuve-Saint-Germain tend donc à situer l'occupation de cet habitat vers les deux derniers tiers du $\mathrm{I}^{\text {er }}$ s. avant J.-C., mais lorsqu'une datation plus précise peut être avancée, ce qui est le cas pour plus de $18 \%$ du corpus, c'est plutôt le troisième quart de ce siècle qui peut être retenu.

\section{Les amphores}

Elles ont fait l'objet d'une étude, parallèlement à celles de Condé-sur-Suippe/Variscourt, dans le cadre d'un mémoire de maîtrise (Hénon, 1991). Dans l'étude morphologique, ce mobilier est divisé en quatre groupes en fonction de la hauteur et de l'inclinaison de la lèvre (fig. 28). Les groupes 1 et 2 caractérisent La Tène D1. Ie groupe 1 est absent de Villeneuve-Saint-Germain et le groupe 2 y est peu représenté. En revanche, le groupe 3 


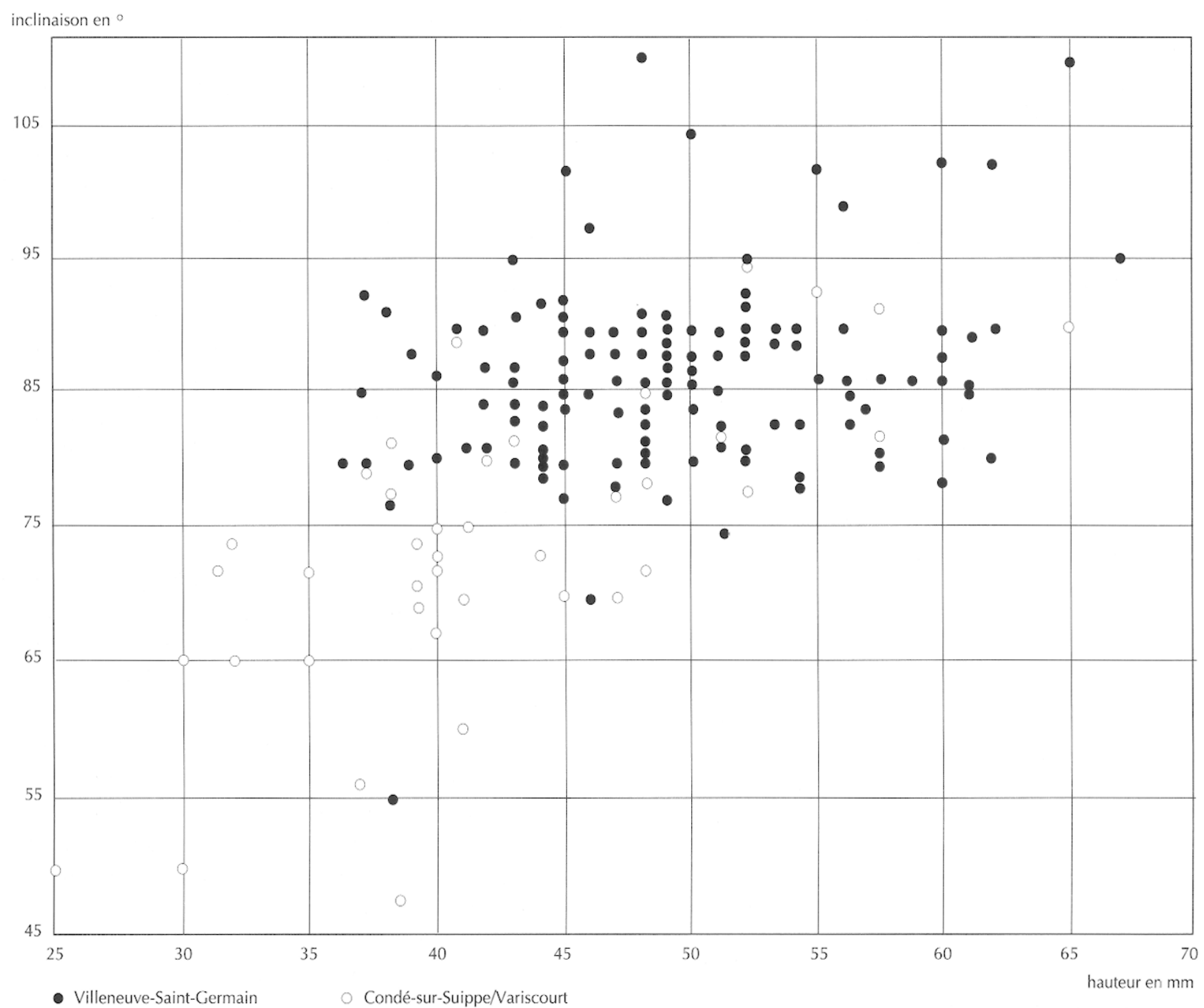

Fig. 29. Évolution des types d'amphores entre Villeneuve-Saint-Germain, Condé-sur-Suippe/Variscourt.

y est largement majoritaire et le groupe 4 est encore présent. Le diagramme (fig. 29) montre bien l'évolution entre les sites de Condé-sur-Suippe et de VilleneuveSaint-Germain, chaque type d'amphore étant progressivement remplacé par un autre. Des schémas d'évolution similaires ont été mis en évidence lors de la périodisation des sites du Forez (Vaginay, Guichard, 1988). Cette première approche des amphores donne donc une datation assez large centrée sur la phase de La Tène D2, datation qui peut être affinée par la présence de vingt-sept estampilles (fig. 30). Si la plupart de ces timbres semblent inédits, certains sont connus sur d'autres gisements dans des contextes bien datés. Ainsi en est-il de la marque M $\left(\mathrm{n}^{\circ} 10\right.$ et 11) qui existe à Roanne en deux exemplaires, l'un provenant d'un contexte $-30 /-20$, l'autre d'un contexte $-50 /-30$ (Aulas, 1980-1981). Le timbre MV $\left(\mathrm{n}^{\circ} 12\right)$ existe également à Roanne dans un contexte $-50 /-30$ de même que le timbre $\mathrm{PH}(\mathrm{IL})\left(\mathrm{n}^{\circ} 19\right)$. Toujours sur le même site, une marque incomplète, semblable à notre $\mathrm{n}^{\circ} 17$ comporte également les deux dernières lettres //AC (Aulas, 1980-1981). Le contexte est $-50 /-30$. La datation de ces estampilles tend donc à resserrer la chronologie vers les années 50/30 avant J.-C. Il faut encore noter la présence de cinq amphores Dressel 2/4, d'une amphore Dressel 20 et d'une Pascual 1. 


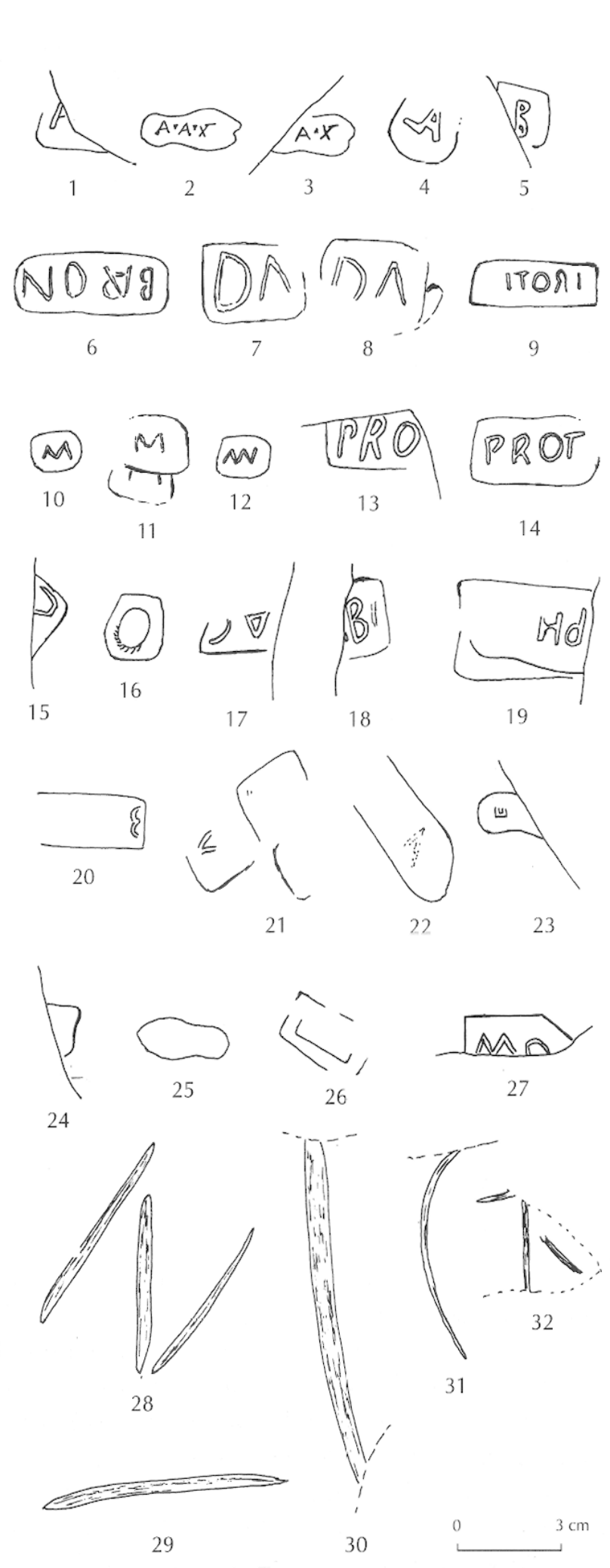

Fig. 30. Estampilles sur les amphores de Villeneuve-Saint-Germain.

\section{Les parures en verre}

Il semble, selon Rupert Gebhard (1989, p. 102), que les contextes datables de La Tène D2 ne comprennent plus de bracelets, mais seulement des perles. Or, le site de Villeneuve-Saint-Germain a livré soixante-quinze perles pour un seul fragment de bracelet, ce qui tend à indiquer, pour notre site, une datation à La Tène $\mathrm{D} 2$. Le bracelet appartient au groupe 3b de Haevernick (1960) dont l'apparition se situe à la Tène C1. Sa présence ici peut signifier, soit que cette forme a perduré jusqu'à La Tène D2, soit qu'il s'agit d'un fragment ancien récupéré pour servir de matière première à l'usage des verriers dont l'existence d'un atelier semble attestée (Debord, 1993b, p. 89).

\section{Les animaux}

Il a été constaté que la taille des animaux, particulièrement des bovidés, va décroissant du Néolithique jusqu'à la période gauloise. Puis, cette évolution s'interrompt brusquement lors de la romanisation (Méniel, 1987, p. 20-23). Or, un accroissement de la taille touche les animaux de Villeneuve-Saint-Germain qui sont tous relativement grands. On constate, par exemple, un net accroissement par rapport à ceux de Condé-sur-Suippe/ Variscourt. Ce phénomène peut donc apparaitre comme un argument très fiable pour avancer une datation postérieure à la conquête.

\section{La cruche de type Ornavasso-Ruvo}

Elle est représentée par une applique d'anse en forme de buste de Jupiter de bonne facture (fig. 31,1 ). Ce type de cruche ${ }^{37}$ est datable de La Tène D2 (Boube, 1991, p. 35).

\section{La céramique}

La céramique dorée au mica

Sa présence est un bon indice de datation tardive puisqu'elle n'est pas connue avant l'époque augustéenne. Elle fait une timide apparition à Villeneuve-Saint-

37. C'est par erreur que ce buste a été répertorié comme appartenant à une cruche bitronconique de type Piatra Neamt dont l'anse se termine toujours par une applique modelée en buste masculin très fruste (Boube, 1991). L'auteur, qui n'avait pas vu l'original, s'est fondé sur une mauvaise photographie (qui plus est, publiée à l'envers) parue dans un ouvrage collectif (Archéologie d'une vallée, La vallée de l'Aisne des dermiers chasseurs-cueilleurs au premier royaume de France, Soissons, 1991). 
Germain avant d'atteindre une bonne représentation dans les couches les plus anciennes d'Augusta Suessionum, l'actuel Soissons (Ancien, Tuffreau-Libre, 1980).

\section{Le décor ondé incisé au peigne}

Il apparaît fréquemment et peut être considéré comme un bon marqueur chronologique caractéristique de la fin de La Tène et du début de la période augustéenne. Michel Perrin (1974, p. 126) note que ce décor, qui se caractérise par plusieurs traits parallèles, n'apparaît que tardivement, "dans les couches les plus récentes, à une période que l'on peut situer vers 30 avant J.-C. ». Anne Colin (1990) en fait un décor caractéristique de la phase III de sa chronologie des oppida, laquelle s'intègre parfaitement dans la séquence de La Tène D2.

\section{L.es dolia}

Comme les amphores, les dolia de Villeneuve-SaintGermain viennent de faire l'objet d'un mémoire (Gransar, 1991). Bien que l'évolution du dolium soit la moins rapide et la moins sensible parmi toutes les poteries, son étude peut donner une indication chronologique. Elle a porté sur 16999 fragments provenant de quatre cent soixante-cinq fosses et correspondant à cinq cent huit individus. Après comparaison avec des dolia issus de corpus diachroniques provenant d'autres sites bien datés, l'auteur distingue deux groupes, l'un, de faible importance, pouvant correspondre à la transition LT Dl/D2 l'autre, nettement plus important, s'intégrant à l'horizon La Tène D2 (p. 119).

\section{Le façonnage au tour}

Le pourcentage relativement important de céramique tournée semble bien, lui aussi, témoigner de l'évolution terminale de La Tène après la conquête, l'usage du tour ne s'étant développé que tardivement dans nos régions.

\section{Autres objets}

L'umbo de bouclier (fig. 31, 2)

Cet $u m b o$ à ailettes trapézoïdales très évasées appartient au type $\mathrm{V}$ C d'André Rapin qui le situe à la dernière phase de la civilisation celtique (Bruneaux, Rapin, 1988, p. 81 et n. 67, p. 76$)$.

La lampe à huile (fig. 31, 3)

C'est une lampe à réservoir ouvert, avec une perforation centrale permettant sa fixation sur un support. Il s'agit
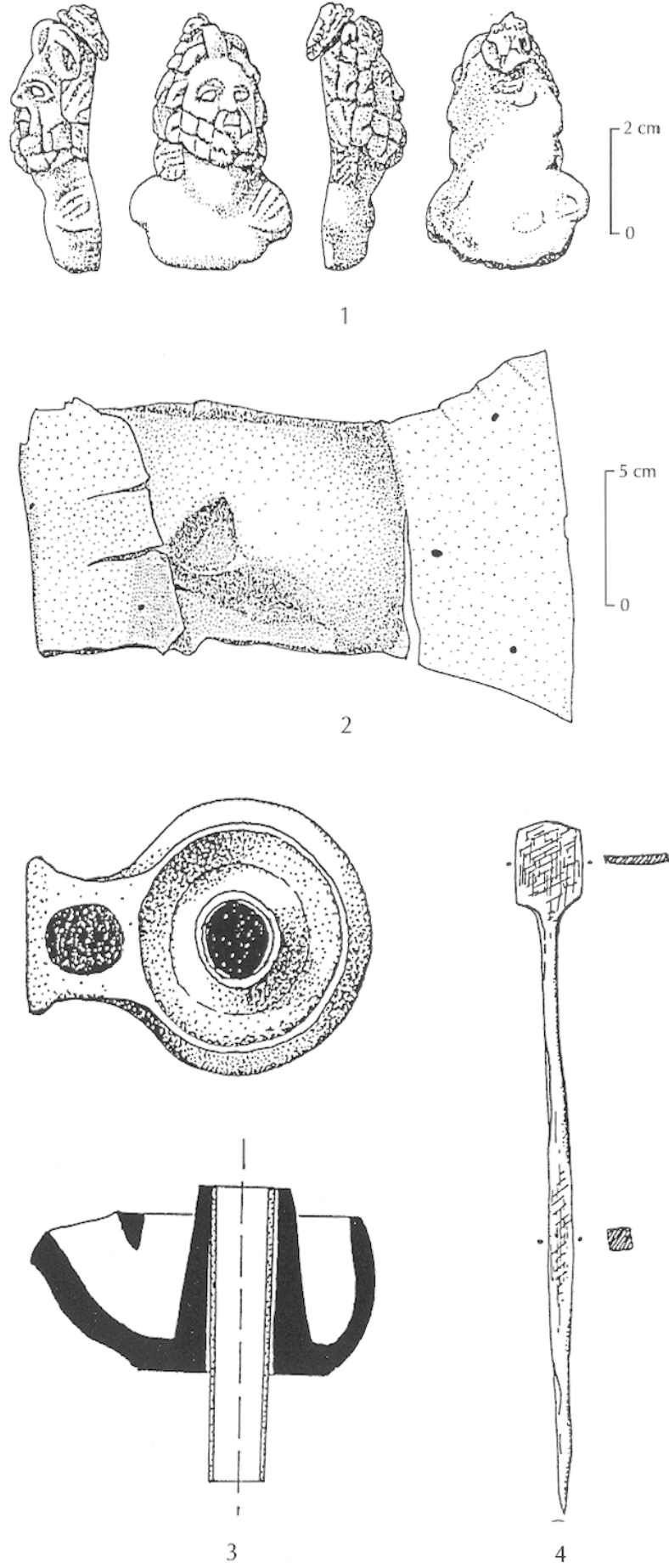

Fig. 31. Quelques traceurs chronologiques pour le site.

1. applique d'anse de cruche;

2. umbo de bouclier;

3. lampe à huile;

4. stylet. 
d'un modèle d'importation italique dont on peut trouver des échantillons aux $\mathrm{II}^{\mathrm{e}}$ et $\mathrm{I}^{\mathrm{er}}$ s. avant J.-C., mais qui paraît dérivé d'un type grec beaucoup plus ancien, à bec arrondi, dont il existe des exemples à partir du V $\mathrm{V}^{\mathrm{e}} \mathrm{s} .{ }^{38}$. En Gaule septentrionale, un exemplaire très proche $\mathrm{du}$ nôtre provient de la tombe aristocratique de Clémency, au grand duché de Luxembourg (Metzler et alii, 1991, p. 46, 75 et 77). Ce modèle de création nettement antérieure à la conquête et dont la production s'est poursuivie sur une longue période ne semble donc pas être un indice très valable pour dater notre site. Mais sa présence sur un habitat en Gaule Belgique peut constituer un argument de datation tardive, l'usage d'un tel objet dans cette région étant difficile à concevoir antérieurement à la présence romaine.

Le stylet (fig. 31, 4)

C'est le même raisonnement (voir ci-dessus) qui nous incite à considérer comme postérieur à la conquête l'utilisation d'un stylet destiné à l'écriture sur des tablettes de cire.

\section{Les monnaies}

Certaines de nos monnaies contiennent en elles-mêmes leur élément de datation. En effet, sur la plupart des monnaies en argent issues de l'atelier monétaire, des traces de surfrappe ont été relevées et plusieurs des types sousjacents, ayant servi de support à la nouvelle image monétaire, ont pu être identifiés. Ce sont, notamment, la monnaie à la légende $Q$. DOCI SAM $F$, la monnaie à la légende KALETEDV, celle à la légende SEQUANOIOTVOS, les types BN 4097 et 5138 . Il s'agit donc de deniers gaulois provenant de diverses régions de Gaule et dont la plupart étaient présents dans les fossés d'Alésia et circulaient donc encore en -52. Leur réutilisation pour servir à la frappe de nouveaux types suppose qu'ils n'étaient plus en circulation et indique une fabrication tardive postérieure à la conquête. D'autre part, le statère « British $Q$ » est une imitation du statère anépigraphe des Suessiones dont nous avons vu qu'il peut être daté du début de la guerre des Gaules. L'imitation britannique est donc plus tardive. D.F. Allen (1961, p. 128-129) situe sa production vers $-40 /-20^{39}$.

\footnotetext{
38. Nous remercions Colette Bémont pour l'aide qu'elle a bien voulu nous apporter dans la recherche de la documentation relative à cet objet. 39. Notons, toutefois, que certains auteurs remettent en question l'origine insulaire de cette monnaie qui pourrait avoir été importée du continent (voir notamment : Fitzpatrick, Megaw, 1987; Fitzpatrick,
}

En conclusion, l'ensemble des éléments que nous venons d'examiner semble donc s'intégrer dans l'horizon I.a Tène D2. Le mobilier pouvant être daté avec plus de précision indique les périodes $-50 /-30,-30 /-20$ ou le troisième quart du $\mathrm{I}^{\mathrm{cr}} \mathrm{s}$. avant $\mathrm{J}$.-C. L'absence totale de céramique sigillée après douze années de fouilles semble significative et incite à placer l'abandon du site vers les années $-20 /-15$. La fourchette d'occupation du site peut donc être estimée raisonnablement à $50 / 15$ avant J.-C. environ. La présence, en faible quantité, de mobilier plus ancien suggère que les habitants se sont installés ici avec leurs objets. On pourrait toutefois admettre, mais avec moins de probabilité nous semble-t-il, l'existence d'un petit noyau plus ancien. Mais cela ne semble pas apparaitre actuellement dans la répartition spatiale du matériel.

Si la datation relative de Villeneuve-Saint-Germain à La Tène D2 semble unanimement admise, il n'en va pas de même pour la datation absolue que nous placions après la conquête et que nos deux collègues, Patrick Pion et Vincent Guichard ${ }^{40}$, lors d'une intervention orale à cette table ronde, situent antérieurement aux campagnes de César, s'appuyant pour ce faire sur une comparaison entre les mobiliers issus de Villeneuve-Saint-Germain et de Pommiers (l'antique Noviodunum), les deux gisements n'étant distants que de $5 \mathrm{~km}$ environ. Cette hypothèse impliquerait un remaniement de la chronologie laténienne puisque La Tène $\mathrm{D} 2$ serait à remonter dans sa totalité avant la guerre des Gaules; elle inverserait, en outre, le schéma que nous avions établi d'un déperchement par-

1992). D'autre part, Van Arsdell (1989) propose une datation un peu plus haute que celle avancée par Allen, soit 55-45 avant J.C.

40. V. Guichard, intervention orale lors de la table ronde de février 1992 : "Plusieurs programmes régionaux de longue haleine ont permis de rassembler au cours des deux dernières décennies des collections cohérentes de monnaies laténiennes, provenant de sites bien stratifiés ou, mieux encore, à occupation courte. Plus précisément, l'examen conjoint des séries livrées par le Forez (Loire), les Limagnes d'Auvergne (Puy-deDôme) et la vallée de l'Aisne (Aisne et Ardennes) nous semble permettre de proposer un nouveau cadre d'évolution de l'utilisation de la monnaie de bronze en Gaule chevelue depuis son introduction jusqu'à la mise en place de l'atelier impérial de Lyon, à la fin du I ${ }^{\text {er }}$ s. avant J-C. Il permet de reconsidérer certaines caractéristiques de la circulation monétaire en Gaule chevelue et la chronologie tant absolue que relative de certains sites dont Pommiers et Villeneuve-Saint-Germain. "

Cf. : GUICIAARD V., PION P., MAIACHFR F., COLLIS J., à paraître : À propos de la circulation monétaire en Gaule chevelue aux II et I $^{\text {er }}$ s. avant J.C., article soumis à la Rerne Archéologique du Centre, 1993. - HASELGROVE C.C., 1990, Later Iron Age Settlement in the Aisne Valley : Some Current Problems and Hypotheses, in: Ies âges du Fer en Bretagne, $12^{\mathrm{e}}$ colloque de l'AFEAF, Quimper, 1988, Rennes, 1992, p. 249-259 ( $3^{\mathrm{e}}$ suppl. à la $\left.R A O\right)$.

- Mal.acher F., Collis J., Chronology, Production and Distribution of Coins in the Auvergne, in : Mays (dir.), 1992, p. 189-206. 


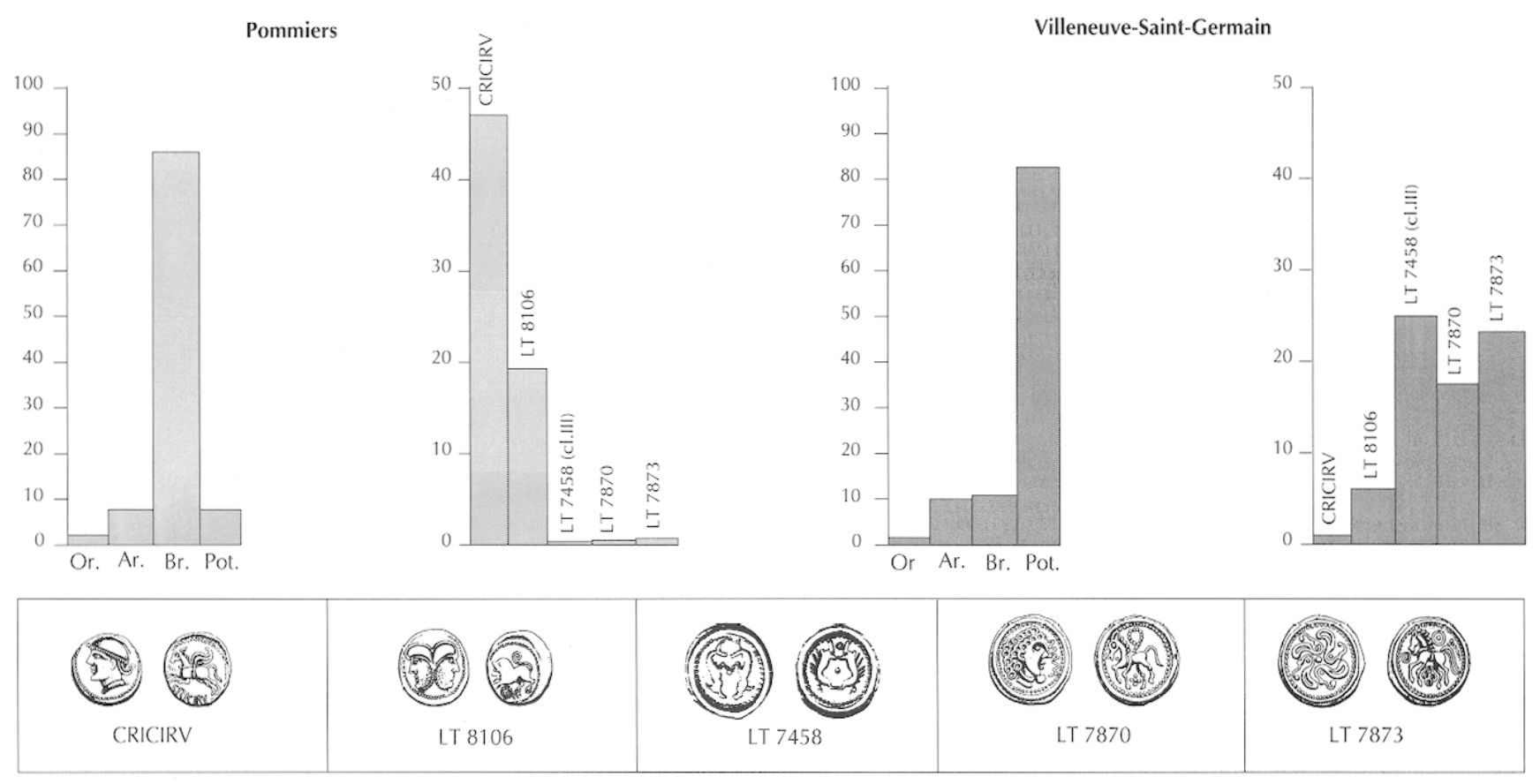

Fig. 32. Faciès monétaires de Pommiers et Villeneuve-Saint-Germain.

tiel de Noviodunum après l'arrivée des Romains (Debord, 1984) ; enfin, elle bouleverserait totalement la vision des numismates puisqu'elle situe en premier l'apparition d'un monnayage en potin qui serait suivi par les émissions en bronze frappé. Pommiers est connu essentiellement par un abondant matériel numismatique (plusieurs milliers de monnaies) provenant, pour la quasi-totalité, de récoltes de surface. I.e matériel issu des fouilles de Vauvillé, au XIX's s., n'est plus exploitable. Restent les fouilles effectuées en 1987 et 1988 par l'ERA 12 du CNRS. En 1987, une coupe a été effectuée à travers le système défensif. Elle a livré trop peu d'objets pour permettre d'avancer une datation. Seules des fosses recoupant le rempart à sa base, donc postérieures à celui-ci, ont livré du matériel céramique d'époque augustéenne. En 1988, la fouille a été prolongée sur $22 \mathrm{~m}$ vers l'intérieur du site, soit sur un peu moins de $150 \mathrm{~m}^{2}$. Il s'agit donc d'un sondage très ponctuel qui ne saurait, en l'état actuel de la recherche, être considéré comme représentatif de l'ensemble de l'oppidum. Dix-sept monnaies y ont été recueillies et publiées (Brun, Debord, 1991). La céramique, encore inédite, vient d'être étudiée par Patrick Pion. Elle semble en majorité augustéenne, ce qui ne doit pas surprendre puisque " les traces de l'occupation augustéenne, denses dans le secteur fouillé, se composent de tranchées de fondation et de fosses profondes (Brun,
Debord, 1991, p. 55) qui ont dû perturber les niveaux sous-jacents ". C'est donc avec la plus grande prudence qu'il convient d'exploiter le mobilier de Pommiers. Toutefois, le faciès monétaire, même s'il résulte d'un mélange de différentes époques, peut être considéré comme représentatif du site, compte tenu du nombre considérable d'individus recueillis. D'autre part, la simple comparaison des populations monétaires de Pommiers et de Villeneuve-Saint-Germain, telle qu'elle apparaît sur les diagrammes (fig. 32), montre à l'évidence le diachronisme des deux habitats avec un chevauchement partiel probable que fait apparaître la présence, sur chacun d'eux, du bronze frappé Scheers 154 (LT 8106). Les monnaies en provenance de l'atelier monétaire de Villeneuve-SaintGermain (Debord, 1989) sont faiblement représentées à Pommiers (trois exemplaires du type Scheers 185, classe III; sept exemplaires du type Scheers 196, classe II; huit exemplaires du type Scheers 197), ce qui pourrait surprendre si l'on considère la proximité des deux sites. Il n'y a cependant pas lieu de s'en étonner. En effet, on constate que les espèces émises à Villeneuve-SaintGermain ne sont jamais représentées par plus de quelques unités sur les autres gisements, même ceux très proches comme Bucy-le-Long ou Condé-sur-Aisne. Il est donc avéré que Pommiers et Villeneuve-Saint-Germain n'ont pas connu leur occupation principale à la même 
époque, que le rempart de Pommiers est antérieur à la période augustéenne, que le matériel augustéen recueilli à Pommiers n'est pas forcément représentatif de l'ensemble du site mais prouve simplement que son occupation a perduré jusqu'à cette période mais sans qu'il soit possible de définir dans quelle proportion. $\mathrm{Si}$, comme nous l'avons montré dans notre exposé, les éléments de datation relative à $\mathrm{La}$ Tène $\mathrm{D} 2$ sont nombreux à Villeneuve-Saint-Germain, il n'en va évidemment pas de même en ce qui concerne les éléments de datation absolue qui semblent pourtant exister. On les trouve d'une part parmi les fibules, d'autre part dans les monnaies. Les fibules dites « à coquille » représentent un pourcentage non négligeable de l'ensemble de ce type de mobilier. Elles se composent de quelques exemplaires classiques en bronze, mais surtout de grandes fibules en fer à arc triangulaire rubané qui s'épanouit en coquille venant protéger le ressort à quatre spires et corde interne. Jusqu'à ce que débutent nos fouilles, ces fibules semblaient peu répandues en France. Un exemplaire en bronze, provenant du fanum de Cracouville à Vieil-Évreux, a été publié par A.-M. Dolfuss (1973, p. 73; p. 76, pl. 6; p. 77, n50). Ce fanum est daté des $\mathrm{I}^{\mathrm{er}}$ et $\mathrm{II}^{\mathrm{e}} \mathrm{s}$. C'est surtout dans les régions de Trèves, de Luxembourg et du bassin de Neuwied que ce type a été rencontré (Mahr, 1967, p. 44; Decker, 1968, p. 43; Haffner, 1977, p. 96, p. 97, fig. 1,2; Riekhoff, 1975, p. 15). Il est apparu au dernier tiers du $\mathrm{I}^{\mathrm{er}} \mathbf{s}$. avant J.-C. et on le trouve jusqu'à la période augustéenne tardive, la datation étant assurée par des mobiliers de sépultures. En ce qui concerne les monnaies, nous avons déjà signalé que la quasi-totalité du monnayage en argent sorti de l'atelier monétaire de Villeneuve-SaintGermain avait été réalisée par la méthode de la surfrappe sur des deniers gaulois qui circulaient encore en -52 puisqu'ils étaient présents dans les fossés d'Alésia. C'est pourquoi nous considérions comme probable sa datation tardive, estimant qu'il était peu vraisemblable que l'on ait utilisé un numéraire toujours en circulation pour la réalisation d'un type nouveau. Mais nous admettrons qu'il ne s'agit que d'une conjecture fondée sur un raisonnement qui nous semble logique mais qui demeure peut-être fort éloigné de la logique des Gaulois! Ce numéraire a peu circulé. Seul le type Scheers 50 (IT 7220) a été trouvé sur d'autres sites (voir fig. 26). Un exemplaire a été trouvé à Meaux, au lieu-dit la Bauve (fouilles de Robert Richard). Il provient des niveaux Auguste/Claude $(-27 /+54)$. Un autre exemplaire a été trouvé en Belgique, à Weyler. Il a été ramassé en surface, au-dessus d'une nécropole augus- téenne (Huysecom, 1982, p. 5). Tant les surfrappes que les découvertes en couches ou en contexte augustéen semblent donc bien indiquer une datation absolue postérieure à la conquête. Quant au statère "British $Q$ ", imitation du statère anépigraphe des Suessiones dont l'émission au début de la guerre semble assurée (Scheers, 1977, p. $60 \mathrm{sq}$.), D.F. Allen situait sa production vers $-40 /-20$. Depuis, certains auteurs ont mis en doute son origine insulaire, estimant qu'il pourrait s'agir d'une production continentale (Fitzpatrick, Megaw, 1987; Fitzpatrick, 1992) et proposent une datation plus haute mais toujours postérieure au commencement de la guerre, soit $-55 /-45$ (Van Arsdell, 1989). Le très fort pourcentage de potins à Villeneuve-Saint-Germain (près de $82 \%$ ) ne doit pas surprendre puisque l'atelier local produisait justement des monnaies en bronze coulé ainsi que l'attestent les restes monétaires trouvés en fouille. Cette forte proportion n'a donc aucune signification sur le plan chronologique et toute comparaison en ce sens avec d'autres sites serait vaine.

Si le site de Villeneuve-Saint-Germain peut être daté postérieurement à la conquête romaine, l'occupation de Pommiers doit être placée avant ces événements, en soulignant toutefois qu'une faible occupation de ce site s'est poursuivie tardivement, parallèlement à celle du site du méandre, les deux habitats ayant été abandonnés, en quasi-totalité après la construction d'Augusta Suessionum au croisement des voies romaines. Rien, actuellement, ne nous semble devoir remettre en cause le schéma que nous avions établi antérieurement (Debord, 1984). Dans l'attente de recherches plus étendues, en divers points de l'oppidum de Pommiers, qui s'étend sur 40 ha, il nous semble imprudent de vouloir actuellement avancer une hypothèse qui, outre la remise en question des chronologies relatives des deux sites de Pommiers et de VilleneuveSaint-Germain, tend à déplacer la chronologie de La Tène dans son ensemble.

Jean DEBORD 
Tabl. 4. Inventaire des monnaies appartenant aux types monétaires issus de l'atelier de Villeneuve-Saint-Germain.

\begin{tabular}{|c|c|c|c|c|c|c|c|c|c|c|}
\hline \multirow{2}{*}{ Sites } & \multicolumn{9}{|c|}{ Type } & \multirow{2}{*}{ Bibliographie } \\
\hline & S 185 & S 188 & S 196 & S 197 & S 189 & S 50 & $\begin{array}{c}\text { au } \\
\text { bige }\end{array}$ & $\begin{array}{l}\text { fruste } \\
\text { natté }\end{array}$ & S $30 / 1$ & \\
\hline & $\mathrm{Cl} . \mathrm{III}$ & & Cl. II & & & & & & & \\
\hline Ambleny & & & & 1 & & 1 & & & & Scheers, 1977, p. 492 et 776 \\
\hline Arcy-Saint-Restitue & & & & & 1 & & & & & Scheers, 1977, p. 753 \\
\hline Azy-sur-Marne & & & 2 & 1 & & & & & & Scheers, 1977 , p. 773 et 776 \\
\hline Beaumont-sur-Oise & & & & 1 & & & & & & Scheers, 1977, p. 778 \\
\hline Beauvais & & & & 1 & & & & & & Woimant, 1990, p. 79 \\
\hline Béthisy-Saint-Martin & & & 1 & & & & & & & Jouve, 1981, p. 3 \\
\hline Bucy-le-Long & 1 & & 1 & 1 & & & & & & inédit, prospections Ph. Féry et fouilles ERA 12 \\
\hline Chilly & 1 & & & & & & & & & Scheers, 1982, p. 100 \\
\hline Cierges & & & & 2 & & & & & & Scheers, 1977, p. 776 \\
\hline Cœeuvres-et-Valsery & & & 1 & & & & & & & Scheers, 1977 , p. 774-775 \\
\hline Coincy & & & 1 & & & & & & & Scheers, 1977, p. 774 \\
\hline Compiègne & & & 2 & & & & & & & Scheers, 1977, p. 775 \\
\hline Condé-sur-Aisne & 1 & & 4 & 1 & & & & & & Scheers, 1977, p. 732, 774, 777 \\
\hline Creil-sur-Mer & & & 1 & & & & & & & Scheers, 1977, p. 776 \\
\hline Crouttes & & & & 1 & & & & & & Scheers, 1977, p. 777 \\
\hline Fère-en-Tardenois & & & & & 1 & & & & & Scheers, 1977, p. 733 \\
\hline Forêt de Compiègne & 14 & 1 & 3 & 3 & & & & & & Scheers, 1977, p. $711,733,775,777$ \\
\hline Great Chesterfield & 1 & & & & & & & & & Scheers, 1977, p. 712 \\
\hline Le Plesier-Huleu & & & 2 & & & & & & & Scheers, 1977, p. 732 \\
\hline Maulévrier & & & 1 & & & & & & & Scheers, 1977, p. 776 \\
\hline Meaux & & 3 & & & 2 & 1 & & & & Scheers, 1977 , p. 733 et 734 ; inédit, fouilles R. Richard \\
\hline Parcy-et-Tigny & & & & 1 & & & & & & Scheers, 1977, p. 777 \\
\hline Paris & & & & 1 & 1 & & & & & Scheers, 1977, p. 733 et 778 \\
\hline Pasly & & & & 1 & & & & & & Scheers, 1977, p. 777 \\
\hline Pierrefonds & & & & 1 & & 1 & & & & Scheers, 1977, p. 775 et 778 ; inédit, M. Dhénin \\
\hline Pommiers & 1 & 1 & 7 & 8 & & & & & & $\begin{array}{l}\text { Scheers, } 1977 \text {, p. } 775 \text { et } 777 \text {; Brun, Debord, 1991, p 49; } \\
\text { Debord, Nominé, 1983, p. } 216\end{array}$ \\
\hline Pont-Sainte-Maxence & 1 & & & & & & & & & Huysecom, 1985, p. 6 \\
\hline Rollot & & & & 1 & & & & & & Scheers, 1977, p. 778 \\
\hline Saint-Martin-Longueau & 1 & & & 1 & & & & & & Scheers, 1977 , p. 778 ; Huysecom, 1985, p. 6 \\
\hline Saint-Thomas & 1 & 1 & & & & & & & & inédit, prospection Y. Nase \\
\hline Senlis & & & 1 & 1 & & & & & & Scheers, 1977, p. 775 et 778 \\
\hline Vendeuil-Caply & 3 & 1 & & 4 & & & & & & Scheers, 1977, p. 711, 733, 778 ; Delestrée, 1985, p. 56 \\
\hline Venizel & & & 1 & & & & & & & Scheers, 1977, p. 775 \\
\hline Vermand & 1 & & & 1 & & & & & & Scheers, 1977, p. $710 ; 1980$, p. 108 \\
\hline Verneuil-en-Halatte & & & 1 & & & & & & & Huysecom, 1985, p. 7 \\
\hline Vieux-Moulin & & & 1 & & & & & & & Scheers, 1977, p. 775 \\
\hline Villeneuve-au-Chatelot & & 1 & & & & & & & & Piette,1987, p. 227 \\
\hline Villeneuve-Saint-Germain & 183 & 9 & 129 & 172 & 1 & 21 & 13 & 1 & 20 & Debord, 1987 a, p. 122-123 \\
\hline Villers-Agron-Aiguizy & & & & 1 & & & & & & Scheers, 1977, p. 777 \\
\hline Weyler & & & & & & 1 & & & & Huysecom, 1982, p. 5 \\
\hline
\end{tabular}




\section{Bibliographie}

\section{ANCIEN B., TUFFREAU-LIBRE M.}

1980, Soissons gallo-romain. Découvertes anciennes et récentes, Soissons, Musée de Soissons.

\section{Aulas CH.}

1980-1981, Marques et inscriptions sur amphores trouvées à Roanne, Cahiers Archéologiques de la Loire, 1, p. 60-91.

BOUBE CH.

1991, Les cruches, in: La vaisselle tardo-républicaine en bronze, Dijon, Université de Bourgogne (Centre de recherches sur les techniques gréco-romaines, 13).

BRUN P., DEBORD J.

1991, Monnaies trouvées en fouilles sur l'oppidum de Pommiers (Aisne), Reoue Numismatique, V'e série, XXXIII, p. $43-59$, pl. IV.

BRUNAUX J.-L., RAPIN A.

1988, Gournay II - Boucliers et lances (A. Rapin) ; Dépôts et trophées (J.-L. Brunaux), Paris, 1988 (Revue Archéologique de Picardie, numéro spécial).

\section{CHAPOTAT G.}

1970, Vienne gauloise. Le matériel de La Tène III trouvé sur la colline Sainte-Blandine, Lyon.

\section{COLIN A.}

1990, La chronologie des oppida en France non-méditerranéenne, in: Les Gaulois d'Armorique, La fin de l'Âge du Fer en Europe tempérée, Actes du XIIe colloque de l'AFEAF, Quimper, mai 1988, Rennes, p. 195-207 (3e suppl. à la $R A O$ ).

\section{DEBORD J.}

1980, L'apport du site de Villeneuve-Saint-Germain (Aisne) à la numismatique - Découvertes récentes, Cahiers Numismatiques, 64, p. 37-55.

1984, Les origines gauloises de Soissons - Oscillation d'un site urbain, in : Les villes de la Gaule Belgique au Haut Empire, Actes du colloque tenu à Saint-Riquier (Somme) les 22, 23, 24 octobre 1982, Revue Archéologique de Picardie, 3-4, p. 27-40.

1985, Un statère anépigraphe des Suessiones découvert à Berzy-le-Sec (Aisne), Revue Archéologique de Picardie, 1-2, p. 21-24.

1987 a, Les monnaies gauloises issues des fossés de partition de l'oppidum de Villeneuve-Saint-Germain (Aisne), in : Brunaux, Gruel, 1987, p. 90-123.

1987 b, Une production tardive en argent de l'atelier monétaire gaulois de Villeneuve-Saint-Germain (Aisne), in : Mélanges offerts au Docteur Colbert de Beaulieu, Paris, p. 235-252.
1989, L'atelier monétaire gaulois de Villeneuve-Saint-Germain (Aisne) et sa production, Revue Numismatique, $6^{\mathrm{e}}$ série, XXXI, 1989, pl. I.

1993 a, Figuration d'une légende de la mythologie germanique sur une monnaie gauloise en potin, in : Actes $d u X I^{e}$ Congrès International de Numismatique, p. 37-42.

$1993 \mathrm{~b}$, Les artisans gaulois de Villeneneuve-Saint-Germain (Aisne) - Structures, production, occupation du sol, Revue Archéologique de Picardie, 3-4, p. 71-110.

DEBORD J., ILETT-FLEURY B.

1983, Attribution de la monnaie gauloise en potin du type BN 7602-7605, Cahiers Numismatiques, 75, p. 211-225.

DEBORD J., NOMINE C.

1983, Nouvel additif aux découvertes monétaires à l'oppidum de Pommiers (Aisne), Cahiers Numismatiques, 75, p. 211-225.

DECKER K.V.

1968, Die jüngere Latènezeit im Neuwieder Becken, Jahrbuch für Geschichte und Kunst des Mittelrheins, 1, Neuweid.

\section{DELESTRÉE L.-P.}

1985, Les monnaies gauloises du temple des « Chatelets " à Vendeuil-Caply (Oise), Revue Archéologique de Picardie, 1-2, p. $51-64$.

\section{Dollfus M.-A.}

1973, Calalogue des fibules de bronze de Haute-Normandie, extraits des Mémoires présentés par divers savants à l'Académie des Inscriptions et Belles-Lettres, Paris.

FEUGÈRE M.

1978, Les fibules du Tournugeois, Bulletin de la Société des Amis des Arts et des Sciences de Toumus, p. 133-198.

1985, Les fibules en Gaule méridionale de la conquête à la fin $d u V^{e}$ siècle après J.-C., Paris, CNRS (12 suppl. à la $R A N)$.

\section{FITZPATRICK A.}

1992, The Roles of Celtic Coinage in South East England, in : Mays (dir.), 1992, p. 1-32.

FiTZPATRICK A., MEGAW J.V.S.

1987, Further Finds from the Le Catillon Hoard, Proceedings of the Prehistoric Society, 53, p. 433-444.

\section{FOUCRAY B.}

1981, Découverte de monnaies gauloises dans la vallée de l'Automne, Reoue Archéologique de l'Oise, 24, p. 11-15, fig. 1-8.

FURGER-GUNTI A.

1979, Die Ausgrabungen im Basler Münster I, Solothurn. 
GEBHARD R.

1989, Le verre à Manching : données chronologiques et apport des analyses, in : Le verre préromain en Europe occidentale, Montagnac.

\section{GRANSAR F.}

1991, Étude des dolia dans un ensemble proto-urbain de La Tène finale: Villeneuve-Saint-Germain (Aisne), Mémoire présenté en vue du diplôme de l'EHESS, École Pratique des Hautes Études en Sciences Sociales, 2 vol.

\section{HAEVERNICK T.E.}

1960, Die Glasarmringe und Ringperlen der Mittel und Spätlatènezeit auf dem europäischen Festland, Bonn.

\section{HAFFNER A.}

1977, Neue Forschungen zur Archäologie der Treverer, Symposium : Ausklang der I atène-7ivilisation und Anfänge der germanischen Besiedlung im mittleren Donaugebiet, Bratislava.

\section{HÉNON B.}

1991, Amphores importées dans la vallée de l'Aisne au Irr siècle avant J.-C. : les oppida de Condé-sur-Suippe, Villeneuve-Saint-Germain, Pommiers, l'établissement rural de Berry-au-Bac, mémoire de maîtrise, université de Paris I, 2 vol.

\section{HUYSECOM E.}

1982, Une monnaie gauloise frappée à Villeneuve-SaintGermain (Aisne) découverte à Weyler (Luxembourg), Bulletin du Cercle d'Études Numismatiques, 19, 1, janvier-mars 1982, p. 5.

1985, Monnaies gauloises récemment mises au jour dans le département de l'Oise, Revue Archéologique de Picardie, 1-2, p. 3-12.

\section{JOUVE $M$.}

1981, Nouvelles découvertes de monnaies aux environs de Compiègne, Reoue Archéologique de l'Oise, 22, p. 2-5.
L.AMBOT B.

1975, Les fibules du musée Vivenel trouvées en forêt de Compiègne sous Napoléon III, Cahiers Archéologiques de Picardie, p. 15-28.

MAHR G.

1967, Die jüngere Latènekultur des Trierer Landes, Berlin.

\section{MÉNIEL P.}

1987, Chasse et élevage chez les Gaulois, Paris, Errance.

\section{PERrin M.}

1974, Techniques de fabrication et de décoration de la céramique antique, Dossiers de l'Archéologie, 6, p. 110-135.

PIETTE J.

1987, Le site des Grèves à La-Villeneuve-au-Châtelot (Aube), note sur quelques ensembles monétaires gaulois, in : Brunaux, Gruel, 1987, p. 211-235.

\section{RIEKHOFF S.}

1975, Münzen und Fibeln aus dem vicus des Kastells Hüfingen, Saalburg Jahrbuch, XXXII, p. 3-104.

\section{SCHEERS S.}

1980, Les monnaies gauloises trouvées à Vermand, Cahiers Archéologiques de Picardie, 7, p. 105-114.

1982, Les monnaies trouvées au fanum de Chilly (Somme) de 1978 à 1980, Revue Archéologique de Picardie, 4, p. 92-118.

\section{VENCLOVA N.}

1989, La parure celtique en verre en Europe centrale, in : Le verre préromain en Europe occidentale, Montagnac, p. 85-97.

\section{WOIMANT G.-P.}

1990, Beauvais, " les Aulnes du Canada " : Viereckschanze ou enceinte quadrangulaire?, Revue Archéologique de Picardie, 34, p. 27-93. 